\title{
UNITARY MEASURES ON LCA GROUPS
}

BY

\section{LAWRENCE CORWIN}

ABSTRACT. A unitary measure on a locally compact Abelian (LCA) group $G$ is a complex measure whose Fourier transform is of absolute value 1 everywhere. The problem of finding all such measures is known to be closely related to that of finding all invertible measures on $G$. In this paper, we find all unitary measures when $G$ is the circle or a discrete group. If $G$ is a torsion-free discrete group, the characterization generalizes a theorem of Bohr.

1. Introduction. Let $G$ be a locally compact group, and let $\mu$ be a finite (complex-valued) regular Borel measure on $G$. Define $\mu^{\sim}$ by $\mu^{\sim}(E)=\overline{\mu\left(E^{-1}\right)}$ for all Borel sets $E$. We call $\mu$ unitary if $\mu^{\sim}=\mu^{-1}$ (i.e., $\mu^{\sim} * \mu=\mu * \mu^{\sim}=\delta_{e}$ ). If $G$ is Abelian, this condition is equivalent to saying that $|\hat{\mu}(\delta)|=1$ for every $\gamma \in \Gamma$, the dual group of $G$.

We investigate the problem of finding all unitary measures on a locally compact Abelian group. The key tools are results of J. Taylor [7] and the Arens-Royden theorem (one form of which is given as Proposition 4.1 of [7]). We obtain complete results when $G$ is discrete (Theorem 5); in the particular case where $G$ is also torsion-free, the answer has a nicer form (Theorem 4), and this result generalizes a theorem of Bohr [1]. We also obtain all unitary measures on the circle group $T$; this was the original aim of the paper. In view of Corollaries 4.6 and 4.7 of [7], these results give characterizations of the measures in $\mathbb{M}(G)^{x}$, the multiplicative group of invertible measures on $G$, when $G$ is one of the above groups.

Before getting down to serious work, we make a few preliminary remarks. If $\mu$ is unitary, write $\mu=\mu_{d}+\mu_{c}$, where $\mu_{d}$ is discrete and $\mu_{c}$ is continuous. Then (see [2]) $\mu_{d}$ is also unitary, and so $\mu=\mu_{d} *\left(\delta_{e}+\mu_{c} * \mu_{d}\right)$. Thus classifying the unitary measures amounts to classifying the discrete ones and those which are (continuous $+\delta_{e}$ ). We call the latter continuous unitary measures.

Next, if $\nu$ is a measure on $\nu$ satisfying $\nu^{\sim}=-\nu$ (for Abelian $G$, this $\Leftrightarrow \hat{\nu}$ is purely imaginary), then $\exp \nu=\delta_{e}+\nu+(\nu * \nu) / 2 !+\cdots$ is unitary. (Conversely, if $\exp \nu$ is unitary, then $\nu^{\sim}=-\nu_{0}$ ) Unitary measures of this form are precisely the ones lying in the connected component of the identity of $M(G)^{x}$. We gener-

Received by the editors February 18, 1972 and, in revised form, July 6, 1973.

AMS (MOS) subject classifications (1970). Primary 43A10, 43A25, 43A70, $22 \mathrm{~B} 99$.

Key words and phrases. Invertible measure, locally compact Abelian group, Fourier transform. 
ally regard these measures as trivial and look for others. Corollaries 4.6 and 4.7 of [7] show that if $\mu \in \mathbb{M}(G)^{x}$, then 3 a measure $\nu_{0} \in M(G)$ such that $\left(\exp \nu_{0}\right)(y)$ $=|\mu(y)|, \forall y \in \Gamma$; it is this result that makes the problem of classifying unitary measures equivalent to the problem of classifying elements of $\pi(G)^{x}$.

I am indebted to the referee for many helpful comments and suggestions. I would also like to thank Professors W. Massey and A. Gleason for some useful conversations.

2. Continuous unitary measures. The main purpose of this section is to prove the following theorem for $T$.

Theorem 1. If $\mu$ is a continuous unitary measure on $T, \mu=\exp \nu$ for some continuous $u$.

Proof. Theorem 3 of [7] says that there are measures $\mu_{1}, \ldots, \mu_{n}, \nu_{0} \in \mathbb{N}(T)$, topologies $\mathfrak{T}_{1}, \ldots, \mathfrak{T}_{n}$ on $T$ (all at least as fine as the usual topology, and all making $T$ into a locally compact group), and complex numbers $\lambda_{1}, \cdots, \lambda_{n}$ such that $\mu_{j}-\lambda_{j} \delta_{e}$ is absolutely continuous with respect to Haar measure on $\left(T, \mathfrak{I}_{j}\right)$ and $\mu=\mu_{1} * \cdots * \mu_{n} * \exp \left(\nu_{0}\right)$. But the only topologies on $T$ satisfying the condition are the usual one and the discrete topology. $\left({ }^{1}\right)$ Hence $\mu=\mu_{1} * \mu_{2} * \exp \left(\nu_{0}\right)$, where $\mu_{1}$ is discrete and $\mu_{2}-\lambda_{2} \delta_{e}$ is absolutely continuous. Write $\nu_{0}=\nu_{1}+\nu_{2}$, where $\nu_{1}$ is discrete and $\nu_{2}$ is continuous; then $\mu=\left(\mu_{1} * \exp \nu_{1}\right) *\left(\mu_{2} * \exp \nu_{2}\right)$; the first expression in parentheses is a discrete measure, and the second is (except for a multiple of $\delta_{e}$ ) continuous. As $\mu-\delta_{e}$ is continuous, the first term must be a multiple of $\delta_{e}$.

We have reduced the theorem to the following: If $\mu$ is unitary and $\mu-\delta_{e}$ is absolutely continuous, then $\mu=\exp \nu$ for some continuous $u_{\text {. In fact, one can }}$ even pick $\nu$ absolutely continuous, as we shall see. We work in $\mathfrak{L}^{1}(T) \oplus \mathrm{C} \delta_{e}$; it suffices to show that $\mu$ is in the connected component of $\delta_{e}$ in $\pi(T)^{x}$. If $\tilde{\mu}(m)$ $\neq-1, \forall m$, then $\delta_{e}+t\left(\mu-\delta_{e}\right)$ never has zero transform, and hence is invertible for all $t$. In general, $\lim _{k \rightarrow \infty} \hat{\mu}_{0}(k)=0$; hence $\hat{\mu}$ is -1 on a finite set. Suppose $\hat{\mu}(k)=-1$ for $k=m_{1}, \cdots, m_{l}$; let $\sigma$ be the measure given by $d \sigma(z)=i\left(z^{m_{1}}+\cdots+z^{m} l\right) d z$ $(d z=$ Harr measure $)$. Then $(\mu+t \sigma)^{\wedge}(k)=\hat{\mu}(k)$ except for $k=m_{1}, \cdots, m_{l}$; at those points, $(\mu+t \sigma)^{\wedge}(k)=-1+i t$. Hence $\mu$ and $\mu+\sigma$ are connected by a line of invertible measures and, as in the first part, $\mu+\sigma$ is in the connected component of $\delta_{e}$. That proves the theorem.

The last part of the proof gives the following result.

(1) This follows from structure theory. Another proof: If $G=(T, g)$, then the identity map of $G \rightarrow T$ gives (by duality) a dense map of $\mathbf{Z}$ into $\hat{G}$. Hence $\hat{G}$ is monothetic. All monothetic groups are $Z$ or compact [5, Theorem 2.3.2]; hence $G$ is $T$ or discrete. 
Corollary 1. Suppose that $G$ is compact. Then if $\mu=\delta_{e}+\mu_{0}$ is unitary and $\mu_{0}$ is absolutely continuous, $\mu=\exp \nu$ for some absolutely continuous $\nu_{0}$

The argument is the same as above. Suppose $\hat{\mu}=-1$ at $\gamma_{1}, \ldots, \gamma_{l}$; one lets $d o(x)=i\left(\gamma_{1}(x)+\cdots+\gamma_{l}(x)\right) d x$ and reasons the same way. (The set where $\hat{\mu}=-1$ is finite because $\hat{\mu}_{0}$ is 0 at $\infty_{0}$ )

The argument of the first half of the theorem reduces the problem for general Abelian $G$ to finding absolutely continuous unitary measures on various other groups (viz., $G$ with finer topologies). But that still leaves a good deal of work in most cases.

A similar attack does, however, work in at least one other case.

Theorem 2. If $\mu$ is a unitary measure on a discrete torsion group $G$, then $\mu=e^{\nu}$ for some $\nu \in M(G)$.

Proof. Since $|\hat{\mu}|=1$ and $\Gamma$ is totally disconnected, $\hat{\mu}(\gamma)=e^{a(\gamma)}$ for some continuous function $a$. Now the Arens-Royden theorem says that $\mu=\exp (\nu)$ for some $\nu \in \mathbb{M}(G)$.

3. Delta measures; unitary measures on torsion-free discrete groups. Theorem 2 implies that if $z \in T$ is of finite order, then $\delta_{z}$, the point mass at $z$ with mass 1 , is of the form $\exp (\nu)$. Here is a converse.

Theorem 3. If $z \in T$ bas infinite order, then $\delta_{z} \neq \exp (\nu)$ for any measure $u_{0}$

Proof. Let $z=e^{i \theta}$. Then $\delta_{z}^{a}(n)=e^{-i n \theta}$; if, therefore, $\exp (\nu)=\delta_{z}$, then $\hat{\nu}(n)=-i n \theta+2 \pi i k_{n}, k_{n} \in \mathbf{Z}$. Let $K=\|\nu\|$, so that $K \geq\left|2 \pi k_{n}-n \theta\right|$, and let $p$ be an integer $>K$. Let $y=e^{-i \theta / p}$. Then $\exp (\nu / p) * \delta_{y}$ has a Fourier-Stielties transform whose range consists of $p$ th roots of unity. Let the roots be $\omega_{1}, \ldots, \omega_{p}$; then the set $S_{j}$ on which the transform is $\omega_{j}$ is, according to results on idempotent measures (see, e.g., [5, p. $61 \mathrm{ff}$.$] ), a union of arithmetic progressions (with$ finitely many exceptions). The idea in what follows is that the irrationality of $\theta / \pi$ makes it impossible for the $S_{j}$ to be so orderly.

Let $N$ be large enough so that the variations in the progressions have been ironed out by then; pick $S_{j}$ so that it contains an infinite arithmetic progression, with common difference $r$, say. Since $\left|2 \pi k_{n}-n \theta\right|<K$, we have $(n \theta-K) / 2 \pi \leq$ $k_{n} \leq(n \theta+K) / 2 \pi \cdot$

Replace $n$ by $n+m r$; we get

$$
\frac{(n+m r) \theta-K}{2 \pi} \leq k_{n+m r} \leq \frac{(n+m r) \theta+K}{2 \pi} \text {, or } \frac{m r \theta-2 K}{2 \pi} \leq k_{n+m r}-k_{n} \leq \frac{m r \theta+2 K}{2 \pi} \text {. }
$$

But $k_{n}-k_{n+m r}$ is a multiple of $p$; thus there is a multiple of $p$ between $(m r \theta-2 K) / 2 \pi$ and $(m r \theta+2 K) / 2 \pi, \forall m$. That means that for all $m$, there is an 
integer between $(m r \theta-2 K) / 2 \pi p$ and $(m r \theta+2 K) / 2 \pi p$, or that $-m r \theta / 2 \pi p$ is congruent $(\bmod 1)$ to a number between $-2 K / 2 \pi p>-1 / \pi$ and $2 K / 2 \pi p<1 / \pi$. As $m r \theta / 2 \pi p$ is irrational, this is impossible; in fact, the numbers $-m r \theta / 2 \pi p$ are dense.

Corollary 2. If $G$ is any locally compact group and $x \in G$ bas infinite order, then $\delta_{x}$ is not an exponential.

Proof. We may as well assume that $G$ is discrete, since if $\delta_{x}=\exp \nu$ and $\nu=\nu_{1}+\nu_{2}$, with $\nu_{1}$ discrete and $\nu_{2}$ continuous, then $\delta_{x}=\exp \nu_{1}$ also. As $T$ is divisible, we can extend the map $a: n x \mapsto e^{i n}$ to a homomorphism (also called $a)$ of $G$ into $T$. If $\delta_{x}=\exp (\nu)$, then it is easily checked that $\delta_{\alpha(x)}=\exp \left(a_{x} \nu\right)$, where $a_{x} \nu(E)=\nu\left(a^{-1}(E)\right)$. But Theorem 3 makes this impossible.

Theorem 3 makes it possible to find all the unitary measures on any torsionfree discrete group.

Theorem 4. Let $G$ be discrete and torsion-free. Then every unitary measure $\mu$ on $G$ is of the form $\delta_{x} * \exp \nu$, for some $x \in G$ and some measure $\nu$ on $G$ with $\nu^{\sim}=-\nu$; moreover, $x$ is uniquely determined by $\mu_{\text {. }}$

Proof. It suffices to show that any function $f: \Gamma \rightarrow \mathrm{C}^{x}$ is homotopic to a character $X_{x}: y \mapsto(x, y), x \in G$. For then we can choose $x \in G$ such that $\hat{\mu} \cdot X_{-x}$ is homotopic to the trivial map. Since $X_{-x}=\delta_{-x}^{a}$, we can use ArensRoyden to show that $\mu * \delta_{-x}=\exp (\nu)$ for some $\nu$, and the theorem follows. The uniqueness of $x$ follows from Theorem 3 .

Now we prove the homotopy result. Since $\Gamma$ is compact, we can use StoneWeierstrass to approximate $f$ by a finite linear combination of characters, $f \approx$ $\sum_{j=1}^{n} a_{j} \chi_{x_{j}}=g$, say, so that $\|f-g\|_{\infty}<1 / 2 \inf { }_{\gamma \in \Gamma}|f(\gamma)|_{0}$. Then $f$ and $g$ are homotopic. Let $\Gamma_{0}$ be the intersection of the kernels of the $\chi_{x_{j}}$. Then $g$ is constant on $\Gamma_{0}$-cosets, and therefore we can define $\bar{g}$ on $\Gamma / \Gamma_{0}$ by $\bar{g}\left(x \Gamma_{0}\right)=g(x) .\left(\Gamma / \Gamma_{0}\right)^{\wedge}$ is the group generated by the $x_{j}:$ therefore $\Gamma / \Gamma_{0}$ is isomorphic to a torus. But it is well known (see, e.g., [3, Theorem II. 7.1]) that the characters of $T^{m}$ represent the homotopy classes of maps on $T^{m}$; hence $\tilde{g}$ is homotopic to a character $\bar{\chi}$ of $\Gamma / \Gamma_{0}$. Pull $\tilde{\chi}$ back to $\Gamma$, getting $\chi$; then $g$ and $\chi$ are homotopic, as desired.

$A$ corollary of the proof is

Corollary 3. If $\Gamma$ is a connected compact group, then $H^{1}(\Gamma, \mathrm{Z}) \cong G$. (The cobomology is (̌ech cobomology.)

Proof. From [4], $H^{1}(\Gamma, \mathrm{Z}) \simeq$ group of homotopy classes of maps from $\Gamma$ to $T$. Since $\Gamma$ is connected, $G$ is torsion-free [5, Theorem 2.5.6]; the last part of the above proof does the rest. (The result is dual to one of Steenrod's: $H_{1}(G, T)$ $\simeq G$. See [6, Theorem 15]). 
4. Unitary measures on arbitrary discrete groups and $T$. We have still not solved the problem of finding the unitary measures on $T_{d}$, since $T_{d}$ has torsion elements. The following example shows that we can actually find other unitary measures besides $\delta$-measures $*$ exponentials.

Let $z_{0} \in T$ have infinite order, and let $\mu=1 / 2\left(\delta_{1}+\delta_{z_{0}}+\delta_{-1}-\delta_{-z_{0}}\right)$. Then if $n$ is even, $\hat{\mu}(n)=1 / 2\left(1+z_{0}^{-n}+(-1)^{-n}-\left(-z_{0}\right)^{-n}\right)=1$, while if $n$ is odd, $\hat{\mu}(n)=$ $z_{0}^{-n}$. Suppose now that $\mu=\delta_{z_{1}} * \exp (\nu)$ for some $z \in T$ and some measure $u_{0}$ Then $\mu * \delta_{z_{1}^{-1}}=\exp (\nu)$. Let $\mu_{0}=1 / 2\left(\mu * \delta z_{1}^{-1}\right) *\left(\delta_{1}+\delta_{-1}\right), \mu_{1}=1 / 2(\mu * \delta$
$\left(\delta_{1}-\delta_{-1}\right)$. Then

$$
\mu_{0}+\mu_{1}=\mu * \delta_{z_{1}^{-1}}, \text { and } \hat{\mu}_{0}(n)=\left\{\begin{array}{l}
\left(\mu * \delta z_{1}^{-1}\right) \hat{(n),} n \text { even, } \\
0, n \text { odd. }
\end{array}\right.
$$

Let $a: T \rightarrow T$ take $z \mapsto z^{2}$, and let $\mu_{0}^{*}=\mu \circ a$. Then $\hat{\mu}_{0}^{*}(n)=\hat{\mu}_{0}(2 n)=z_{1}^{2 n}$; also, $\hat{\mu}_{0}(2 n)=\exp \nu(2 n)=\exp \hat{\nu} *(n)$. Hence $\delta_{z \bar{I}^{-2}}=\mu_{0}^{*}=\exp \left(\nu^{*}\right)$. It follows that $z_{1}$ has finite order in $T$.

On the other hand,

$$
\hat{\mu}_{1}(n)=\left\{\begin{array}{l}
\left(\mu * \delta_{z_{1}^{-1}}\right) \hat{(n)}=\left(z_{1} z_{0}^{-1}\right)^{n}, \quad n \text { odd }, \\
0, n \text { even, }
\end{array}\right.
$$

and $z_{2}=z_{1} z_{0}^{-1}$ has infinite order. Also, $\hat{\mu}_{1}(n)=\exp \hat{v}(n)$ whenever $n$ is odd. We can now use the same reasoning as in the proof of Theorem 3 to get a contradiction. Let $z_{2}=e^{i \theta}$, and define $K, p$, and $y$ as in Theorem 3. Then exp $\nu / p$ $* \delta_{y} * 1 / 2\left(\delta_{1}-\delta_{-1}\right)$ has a Fourier-Stieltjes transform whose range consists of pth roots of unity and 0 ; the value is 0 on $2 \mathrm{Z}$. Define the $S_{j}$ as in Theorem 3 , and the rest of the argument in Theorem 3 goes through. It follows that $\mu$ is not a point measure convolved with an exponential.

What this argument says is that Theorem 4 is false for $G=Z \oplus Z / 2 Z$. ( $G$ is embedded in $T$ as $\left.z_{0} Z \oplus\{-1,1\}_{0}\right)$ Then $\Gamma \cong T \oplus \mathrm{Z} / 2 \mathrm{Z} ; \hat{\mu}$ is 1 on one circle and $z$ on the other. This construction generalizes.

Let $G$ be any discrete group, and let $G_{1}$ be a finite subgroup (of order $n$, say). Then $\Gamma_{1}=G_{1}^{\perp}$ is of index $n$; let $\Gamma_{1}, \Gamma_{2}, \ldots, \Gamma_{n}$ be the cosets. Let $\sigma_{1}$, $\sigma_{2}, \ldots, \sigma_{n}$ be the idempotent measures whose Fourier-Stieltjes transforms are the characteristic functions of $\Gamma_{1}, \ldots, \Gamma_{n}$ respectively. Let $x_{1}, \ldots, x_{n}$ be elements of $G$, and let $\nu$ be a measure on $G$ with $\nu^{\sim}=-u_{\text {. Then }} \mu=\left(\sum_{j=1}^{n} \delta_{x_{j}}^{n} * \sigma_{j}\right) *$ $\exp \nu$ is unitary.

Theorem 5. Every unitary measure on a discrete group $G$ arises in this way. 
Proof. Let $\mu_{1}$ be a unitary measure. As in Theorem 3 , it suffices to show that $\hat{\mu}_{1}$ and $\hat{\mu}$ are homotopic for some $\mu=\left(\sum_{j=1}^{n} \delta_{x_{j}} * \sigma_{j}\right) * \exp (\nu)$. Again, as in Theorem $3, \hat{\mu}_{1}$ is homotopic to a linear combination of finitely many characters: $\hat{\mu}_{1} \sim \sum_{j=1}^{m} a_{j} \chi_{y_{j}}=f$, say. Let $\Gamma_{0}$ be the common kernel of $\chi_{y_{1}}, \cdots, \chi_{y_{m}}$; $f$ gives $\bar{f}$ on $\Gamma / \Gamma_{0^{*}}$. We may assume from now on that $\Gamma_{0}=\{1\}$, since from now on everything will be constant on $\Gamma_{0}$-cosets. Note that $f$ is the transform of a measure; thus $f^{-1} \hat{\mu}_{1}=\left(\exp \nu_{0}\right) \hat{\text {. }}$

Given our assumption, $G$ is generated by $y_{1}, \ldots, y_{m}$; hence $G \cong \mathrm{Z}^{k} \oplus G_{1}$, where $G_{1}$ is finite of order $n_{0}$. Thus $\Gamma_{1}, \ldots, \Gamma_{n}$ are $k$-tori. Hence there are elements $x_{1}, \ldots, x_{n}$ such that $\chi_{x_{j}}$ and $f$ are homotopic on $\Gamma_{j}$. It follows that if $\mu_{0}=\sum_{j=1}^{n} \delta_{x_{j}} * \sigma_{j}$, then $\hat{\mu}_{0}^{-1} f$ is homotopic to the trivial map on each component.

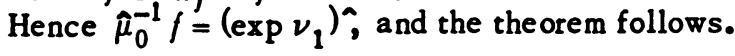

In the case of $T_{d}, G_{1}$ is necessarily cyclic. A more careful analysis along the lines of the example shows that if one picks $m$ as small as possible, then each $x_{j}$ is determined modulo the torsion group of $T_{d}$.

Theorems 1 and 5 together determine all the unitary measures on $T$. As noted earlier, they also determine all the connected components of $M(T)^{x}$. We state the result here for completeness.

Corollary 4. Let $\mu$ be an invertible measure on $T$. Then there are an integer $m$, elements $z_{1}, \ldots, z_{m} \in T$, and a measure $\nu$ on $T$ sucb that $\mu=\exp (\nu) *$ $\left(\Sigma_{j=1}^{m} \delta_{z_{j}} * \sigma_{m, j}\right)$, where $\sigma_{m, j}$ is the idempotent measure on $T$ whose FourierStieltjes transform is 1 on $m \mathrm{Z}+j$ and 0 elsewbere. If $\mu$ is unitary, $\mu$ can be expressed in the same form, but with $\nu^{\sim}=-u$.

\section{BIBLIOGRAPHY}

1. H. Bohr, Über fastperodische ebene Bewegungen, Comment. Math. Helv. 4 (1934), $51-64$.

2. I. Glicksberg, A remark on a theorem of Beurling and Helson, Proc. Amer. Math. Soc. 10 (1959), 587. MR $22 \# 12347$.

3. S. T. Hu, Homotopy theory, Pure and Appl. Math., vol. 8, Academic Press, New York, 1959. MR 21 \#5186.

4. P. Huber, Homotopical cohomology and Čech cohomology, Math. Ann. 144 (1961), 73-76. MR 24 \#A3646.

5. W. Rudin, Fourier analysis on groups, Interscience Tracts in Pure and Appl. Math., no. 12, Interscience, New York, 1962. MR 27 \#2808.

6. N. Steenrod, Universal homology groups, Amer. J. Math. 58 (1936), 661-701.

7. J. Taylor, Inverses, logarithms, and idempotents in $M(G)$, Rocky Mountain J. Math. 2 (1972), no. 2, 183-206. . MR 45 \#5669. 